\title{
Effect of Native Defects on Transport Properties in Non-Stoichiometric $\mathrm{CoSb}_{3}$
}

\author{
Paula R. Realyvázquez-Guevara, Francisco J. Rivera-Gómez, Alejandro Faudoa-Arzate, \\ María E. Botello-Zubiate, Renee J. Sáenz-Hernández, Carlos R. Santillán-Rodríguez and \\ José A. Matutes-Aquino *
}

Centro de Investigación en Materiales Avanzados, S.C., Av. Miguel de Cervantes 120, Complejo Industrial Chihuahua, Chihuahua 31136, Mexico; paula.realyvazquez@cimav.edu.mx (P.R.R.-G.); francisco.rivera@cimav.edu.mx (F.J.R.-G.); alejandro.faudoa@cimav.edu.mx (A.F.-A.); eugenia.botello@cimav.edu.mx (M.E.B.-Z.); joselin.saenz@cimav.edu.mx (R.J.S.-H.); carlos.santillan@cimav.edu.mx (C.R.S.-R.)

* Correspondence: jose.matutes@cimav.edu.mx; Tel.: +52-614-439-1104

Academic Editor: Sofoklis Makridis

Received: 11 January 2017; Accepted: 9 March 2017; Published: 14 March 2017

\begin{abstract}
The effect of native defects originated by a non-stoichiometric variation of composition in $\mathrm{CoSb}_{3}$ on I-V curves and Hall effect was investigated. Hysteretic and a non-linear behavior of the I-V curves at cryogenic temperatures were observed; the non-linear behavior originated from the Poole-Frenkel effect, a field-dependent ionization mechanism that lowers Coulomb barriers and increases emission of charge carriers, and the hysteresis was attributed to the drastic decrease of specific heat which produces Joule heating at cryogenic temperatures. $\mathrm{CoSb}_{3}$ is a narrow gap semiconductor and slight variation in the synthesis process can lead to either $n$ - or $p$-type conduction. The Sb-deficient $\mathrm{CoSb}_{3}$ presented an $n$-type conduction. Using a single parabolic model and assuming only acoustic-phonon scattering the charge transport properties were calculated at $300 \mathrm{~K}$. From this model, a carrier concentration of $1.18 \times 10^{18} \mathrm{~cm}^{-3}$ and a Hall factor of 1.18 were calculated. The low mobility of charge carriers, $19.11 \mathrm{~cm}^{2} / \mathrm{V} \cdot \mathrm{s}$, and the high effective mass of the electrons, $0.66 m_{0}$, caused a high resistivity value of $2.75 \times 10^{-3} \Omega \cdot \mathrm{m}$. The calculated Lorenz factor was $1.50 \times 10^{-8} \mathrm{~V}^{2} / \mathrm{K}^{2}$, which represents a decrease of $38 \%$ over the degenerate limit value $\left(2.44 \times 10^{-8} \mathrm{~V}^{2} / \mathrm{K}^{2}\right)$.
\end{abstract}

Keywords: thermoelectrics; skutterudites; low temperature; electrical transport; native defects

\section{Introduction}

Thermoelectric (TE) materials can generate electric potentials when subjected to a temperature gradient (Seebeck effect) and, conversely, they can transfer heat against a temperature gradient when a current is applied against the generated potential (Peltier effect) [1]. TE materials have attracted great research interest during the past decades due to their potential applications for power generation and are considered as an attractive option for waste heat recovery systems. The main obstacle to widespread use of thermoelectric TE materials is their low efficiency for converting thermal energy into electric energy [2]. The performance of a TE material is related to the dimensionless thermoelectric figure of merit, $Z T$, defined as $Z T=S^{2} \sigma T /\left(\kappa_{\mathrm{e}}+\kappa_{\mathrm{L}}\right)$, where $S$ is the Seebeck coefficient, $T$ is the absolute temperature, $\sigma$ is the electrical conductivity, $\kappa_{\mathrm{e}}$ and $\kappa_{\mathrm{L}}$ are the electronic and lattice thermal conductivities, respectively. The higher the $Z T$, the greater the TE conversion efficiency. But $S, \sigma$ and $\kappa_{\mathrm{e}}$ are entangled, thus, improvement of one parameter usually adversely influences the others [3]. Therefore, high $Z T$ values arise from the beneficial combination of high power factor, $S^{2} \sigma$, and low thermal conductivity, $\left(\kappa_{e}+\kappa_{L}\right)$ [4]. Various approaches have been taken to improve the power factor, 
such as band engineering and electronic state distortion to enhance the Seebeck coefficients $[5,6]$. Thermal conductivity can be lowered by either filling the voids of the crystal structure in TE materials with cage-like structures like skutterudites [7] or by nanopatterning and nanostructuring [8,9].

Skutterudites are good examples of the phonon-glass-electron-crystal (PGEC) concept proposed by Slack [4] in 1995 and, therefore, they are regarded as promising candidates for next-generation TE materials for electrical power generation using either solar energy or waste heat [10]. The general formula of skutterudite compound is $A B_{3}$, where $A$ is a VIIIB group transition metal such as Co, Ir or $\mathrm{Rh}$ and $B$ is a pnictogen atom such as $\mathrm{Ar}, \mathrm{P}$, or $\mathrm{Sb}$. The crystal structure of these compounds belongs to the space group $\operatorname{Im} \overline{3}$ and the unit cell consists of 32 atoms, where six out of the eight simple cubic metal subunits are filled with a near square planar ring formed by four pnictogen atoms $\left(\mathrm{Sb}_{4}\right)$.

Deviation from stoichiometry in skutterudites is a common issue that can result in a large variation in electrical transport properties (i.e., Seebeck coefficient and electrical conductivity) [11]. $\mathrm{CoSb}_{3}$ has been reported with either $p$ - or $n$-type conduction [10-12]. In Sb-rich compositions, a $p$-type conduction has been observed; while in Co-rich compositions, there is a transition from $n$ - to $p$-type conduction above $500 \mathrm{~K}$. Understanding and controlling the native defects originated in non-stoichiometric undoped $\mathrm{CoSb}_{3}$ is essential for the optimization of TE properties of skutterudites.

The influence of $\mathrm{Sb}$ deficiency on electrical transport properties was investigated. The temperature dependence of the I-V characteristics allowed to understand different aspects of the current-transport mechanisms. A shift from linear to non-linear and hysteretic behavior was observed at low temperatures. The non-linear behavior is interpreted on the basis of the appearance of field-dependent mechanisms, which are normally too weak at high temperature. The hysteresis was attributed to the drastic decrease of specific heat at low temperatures which produces Joule heating at cryogenic temperatures. Finally, some charge transport coefficients were calculated in the Sb-deficient sample at $300 \mathrm{~K}$ within the framework of a single parabolic band approximation with a dominant carrier scattering by acoustic phonons. $\mathrm{CoSb}_{3}$ is a narrow-gap semiconductor, and slight variations in non-stoichiometric composition can result in large variations in charge transport properties. In this work, the focus has been to study the influence of native defects on charge transport properties.

\section{Experimental Procedures}

Polycrystalline skutterudite $\left(\mathrm{CoSb}_{3}\right)$ samples were prepared using a sequence of processes, including arc-melting, melt spinning, grinding, cold pressing, and annealing. To avoid oxidation, each individual process was carried out under a protective Ar atmosphere. Co pieces with a purity of 99.5\% (Alfa Aesar, Tewksbury, MA, USA) and Sb shoot with a purity of 99.9999\% (Alfa Aesar) were used as raw materials. $\mathrm{Sb}$ has a low vapor pressure, thus volatilization of $\mathrm{Sb}$ during the fabrication of $\mathrm{CoSb}_{3}$ was compensated by adding $10 \mathrm{wt} \%$ in excess to the stoichiometric amount of $\mathrm{Sb}$. As a first step, an arc melting furnace was used to obtain an ingot. Afterwards, the bulk sample was re-melted under vacuum in an induction furnace and the melted material was ejected onto the copper wheel of a melt-spinning system, rotating with a tangential velocity of $30 \mathrm{~m} / \mathrm{s}$. The resulting product was a mix of finely divided ribbon flakes and wider ribbon sections. The ribbons were grounded using an agate mortar and the resulting powder was loaded into a die cavity of $8 \mathrm{~mm} \times 8 \mathrm{~mm}$. Then, the powder was cold pressed under a uniaxial pressure of $5 \mathrm{MPa}$ during $1.5 \mathrm{~min}$. Finally, the pellet was annealed at $650{ }^{\circ} \mathrm{C}$ for $2 \mathrm{~h}$ with heating and cooling rates of $10^{\circ} \mathrm{C} / \mathrm{min}$.

X-ray diffraction (XRD) phase identification was carried out using a PANalytical X'Pert PRO (PANalytical, Almelo, The Netherlands) with $\mathrm{Cu}-\mathrm{K} \alpha$ radiation. Rietveld refinement was performed using the Fullprof program [13].

The morphology of the ribbons and of the annealed sample were analyzed by scanning electron microscopy (SEM) (JEOL-JSM 5800, JEOL, Tokyo, Japan). Backscattered electron imaging (BEI) and secondary electron imaging (SEI) were employed to observe the details of the microstructure. Chemical composition and sample homogeneity were checked by energy-dispersive X-ray spectrometry (EDS, Tokyo, Japan) microanalysis attached to the SEM. The actual composition was determined by averaging 
the distinct zones on the surface of each sample and by normalizing the resulting chemical formula to one cobalt atom.

$\mathrm{I}-\mathrm{V}$ curves and Hall effect of the annealed prism-shaped sample $\left(8 \times 8 \times 2 \mathrm{~mm}^{3}\right)$ were measured by the AC four-probe method between 2 and $300 \mathrm{~K}$. The sample was soldered to the puck of the AC transport option of a physical property measurement system (PPMS, Quantum Design, San Diego, CA, USA) using four silver wires and $\mathrm{Pb} / \mathrm{Sn}$ braze.

Electrical conductivity, thermal conductivity and Seebeck coefficient were measured between 3 and $400 \mathrm{~K}$ on $14 \times 2 \times 2 \mathrm{~mm}^{3}$ bar samples using the thermal transport option (TTO) of a physical property measurement system (PPMS, Quantum Design). The copper leads were attached to the sample using a two-component silver epoxy (EPOTEK, Billerica, MA, USA). The leads and the epoxy were both provided by Quantum Design.

\section{Results and Discussion}

\subsection{Microstructure}

The X-ray diffraction (XRD) powder pattern of ribbons prepared by melt spinning, is shown in Figure 1a. A complex phase composition with $\mathrm{Sb}, \mathrm{CoSb}, \mathrm{CoSb}_{2}$, and $\mathrm{CoSb}_{3}$ phases were detected, with $\mathrm{Sb}$ as the main phase.

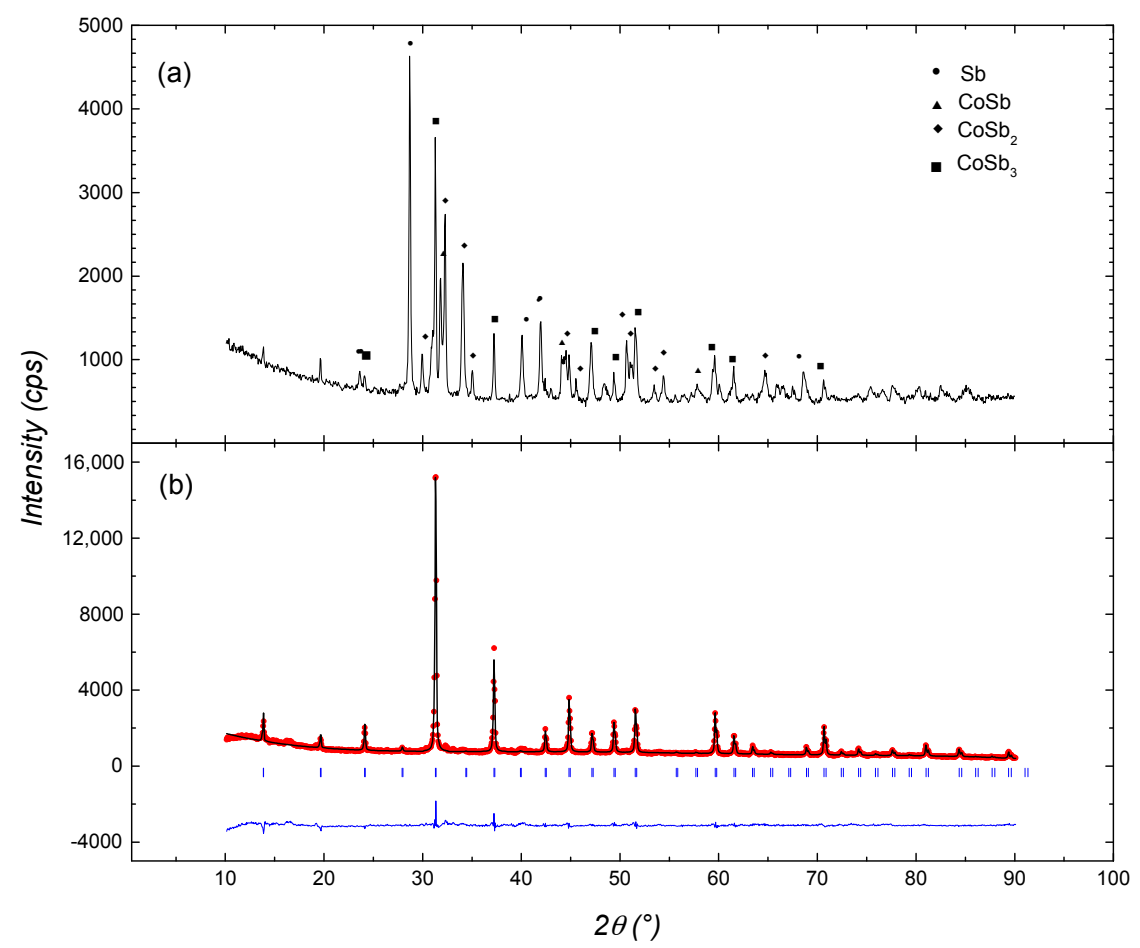

Figure 1. (a) XRD pattern of the ribbons obtained by melt spinning; and (b) Rietveld refinement of the XRD pattern of the annealed bulk sample.

Figure $1 \mathrm{~b}$ shows the Rietveld refinement using the FullProf program for the X-ray diffraction pattern of the annealed bulk sample, where a single-phase sample with a lattice parameter equal to $9.0302 \AA$ was determined.

The melt-spun ribbons have two distinct surfaces: one that directly touches the copper wheel (contact surface) and another which does not contact the copper wheel (free surface). These two sides differ in their microstructure. The microstructure on the contact surface exhibit two different zones, one of them with rounded grains located into depressions with size distribution between 0.5 and $1.25 \mu \mathrm{m}$, and other zone with a mix of rounded, square and irregular grain shapes, with a broader 
size distribution, from 0.1 to $1 \mu \mathrm{m}$, as observed in SEM-BEI image in Figure 2a. The free surface exhibits rounded grains with well-defined grain boundaries, as shown in SEM-BEI images in Figure 2b, with a grain size distribution between 0.85 and $1.5 \mu \mathrm{m}$. These differences in the microstructures of both surfaces were to be expected, since the contacting ribbon surface has a higher cooling rate.

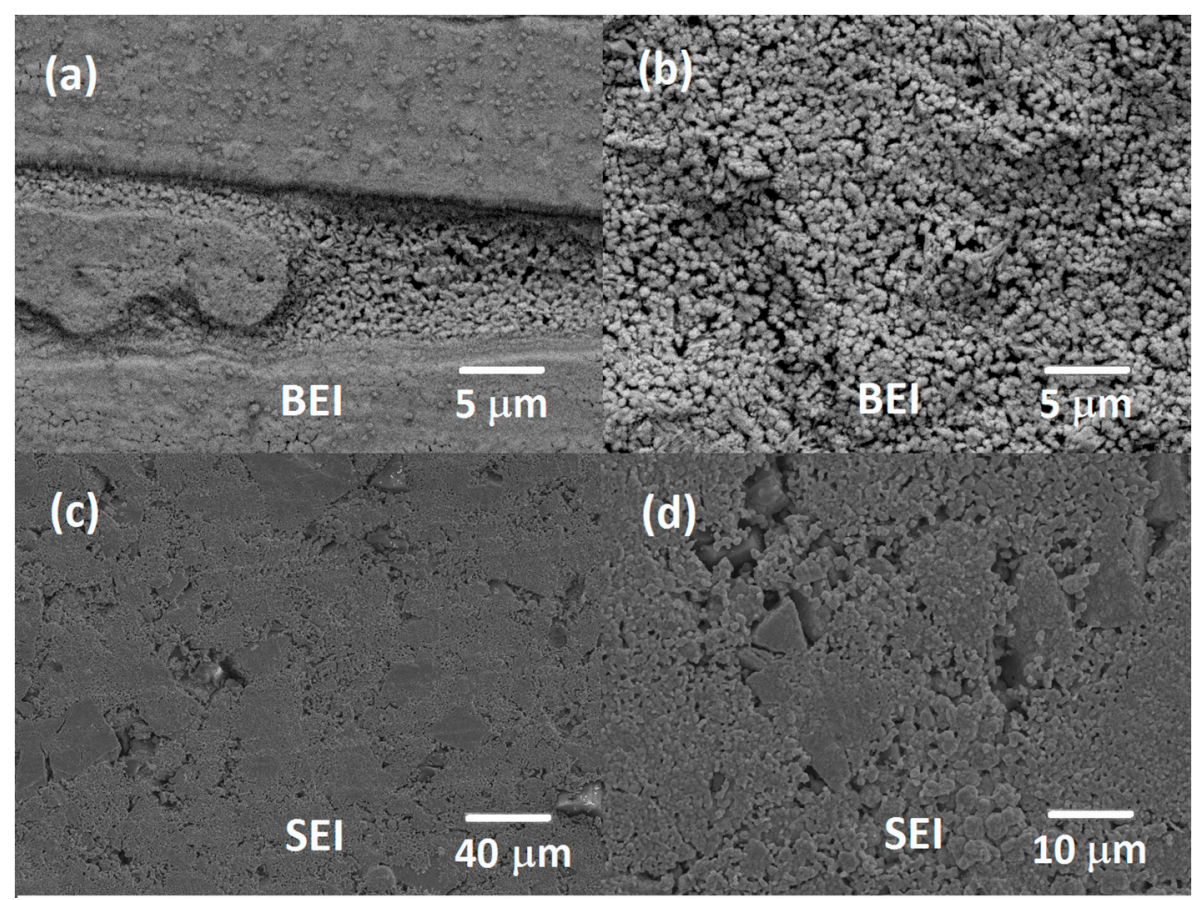

Figure 2. (a) BEI of the contact surface of the ribbon and (b) BEI of the free surface of the ribbon; (c) SEI of the annealed bulk material at $500 \times$ and (d) $5000 \times$.

The annealing of the grounded ribbons resulted in a growth of the grain size, obtaining grains from 0.6 to $50 \mu \mathrm{m}$, as shown in Figure $2 \mathrm{c}$, d.

The elemental composition was determined by averaging 10 separate elemental analyses performed at random zones of the sample. The average atomic percentage of Co was normalized to 1 since it is the least likely element to be lost during processing. The average stoichiometric ratio of $\mathrm{Sb}$ was derived from this normalized value. A nominal composition of $\mathrm{CoSb}_{2.81}$ was determined, which indicates a Sb-deficiency. This deviation from the stoichiometric relation is a very common issue encountered during the fabrication of $\mathrm{CoSb}_{3}$ [14]. The Sb-deficiency provides extrinsic carrier that have impact on the band gap and, hence, on electronic transport properties.

\subsection{Electric Transport Properties}

Figure 3a shows the current-voltage (I-V) characteristics from 2 to $300 \mathrm{~K}$. The I-V curve measured at $2 \mathrm{~K}$ shows a non-linear and hysteretic behavior. I-V measurements taken at closer temperature intervals, between 2 to $40 \mathrm{~K}$, are shown in Figure 3b. Above $T=20 \mathrm{~K}$, the I-V curves are linear, indicating that the resistance is a constant at a given temperature. In other words, the current (I) is proportional to the potential difference $(\mathrm{V})$, resulting in ohmic-like behavior. As the temperature decreases below $T=20 \mathrm{~K}$ a non-linear and hysteretic behavior appears. This behavior becomes more significant as the temperature decreases.

At cryogenic temperatures, electrons are generally trapped in localized states, and they do not have enough energy to get out of its localized state. Although the temperature reduction causes a suppression of the thermal excitation mechanism, which promote electrons from the valence band into the conduction band, the field-dependent ionization mechanism, which is usually too weak at $300 \mathrm{~K}$ to be observed, become important at lower temperatures. Generally speaking, the application of strong 
electric fields enhances emission of carriers due to Poole-Frenkel effect, phonon-assisted tunneling, and direct tunneling. The experimental investigations of carrier emission applying low-electric fields, below $1 \mathrm{kV} / \mathrm{cm}$, which is our case, has been attributed to the Poole-Frenkel effect, which is caused by the lowering of the Coulomb barrier [15].

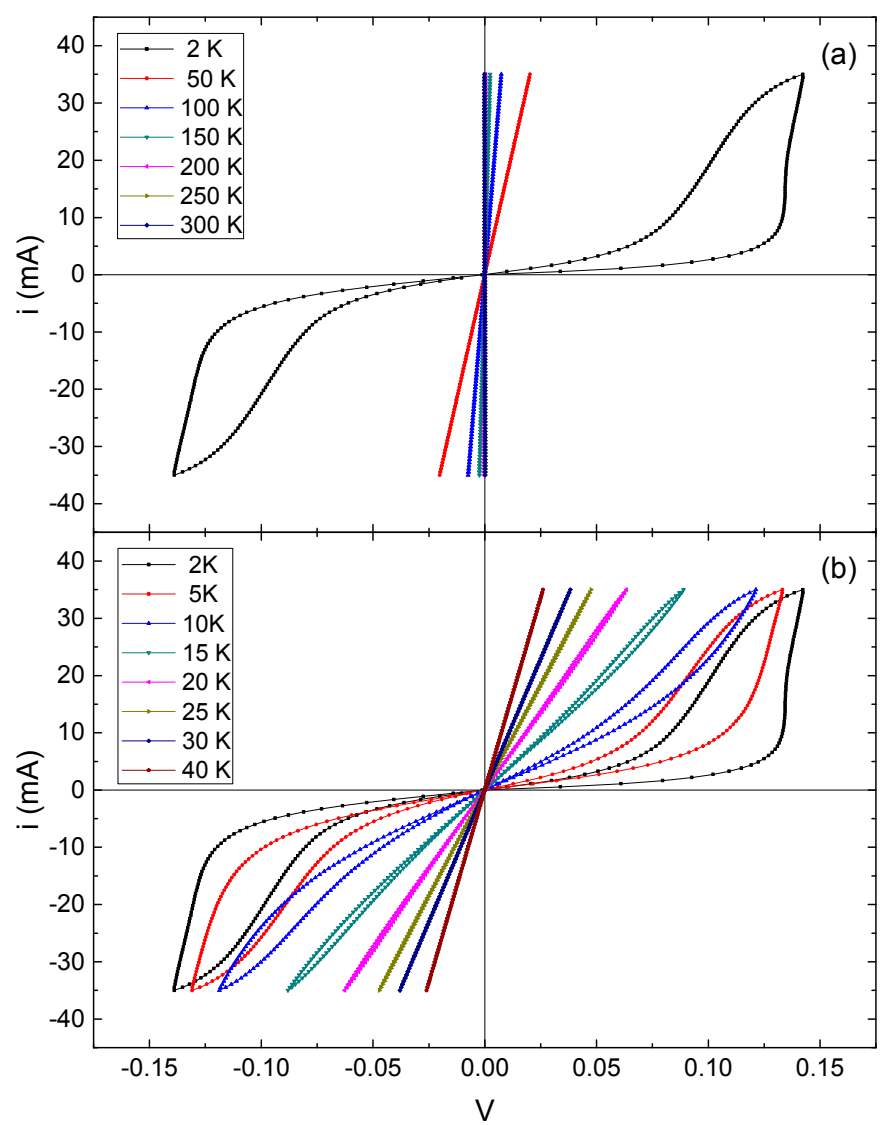

Figure 3. (a) Current-voltage (I-V) characteristics between 2 and $300 \mathrm{~K}$, at $T=2 \mathrm{~K}$ a non-linear and hysteretic behavior appears; and (b) a closer temperature interval, from 2 and $40 \mathrm{~K}$, showed that the non-linear and hysteretic behavior appears at $T<20 \mathrm{~K}$.

As shown in Figure 3b, as temperature decreases and a non-linear behavior appears, there is a critical electric field beyond which the electric current increases more rapidly than in the linear regime. The voltage at which the electric conduction increases more rapidly is known as breakdown voltage and the magnitude of the breakdown voltage in which field-dependent ionization mechanism appears increase as temperature decreases. The effects discussed above disappears at $T>20 \mathrm{~K}$, where thermal energy starts to promote carriers from the valence band into the conduction band (ohmic-like behavior).

Finally, it should be mentioned that, for sufficiently large currents, self-heating of the sample can occur at cryogenic temperatures [16]. This self-heating results from the fact that the specific heat decreases as the temperature is lowered till, as proposed in the Debye model, it approaches to zero at absolute zero. As a result, the lattice temperature may become much higher than the ambient temperature due to Joule heating. This heating changes the charge carrier concentration in the semiconductor, given rise to the hysteretic behavior of the I-V curves.

The temperature dependence of the electrical resistivity of $\mathrm{CoSb}_{2.81}$, Figure $4 \mathrm{a}$, shows that $\rho$ decreases with increasing temperature, which is the classical semiconductor behavior. A value of $2.7 \mathrm{~m} \Omega \cdot \mathrm{m}$ was observed in the sample at $300 \mathrm{~K}$ which is similar to the value reported by other authors [17]. The reported values for $\mathrm{CoSb}_{3}$ resistivity at room temperature differ by three orders of 
magnitude, as reported by Furuyama et al. [18], from $10^{-1}$ to $10^{-4} \Omega \cdot \mathrm{m}$. Samples with Sb-deficiency tends to present higher electrical resistivity, due to changes on the band structure caused by point variations of non-stoichiometry and defect structure [11].

Variations of non-stoichiometry in $\mathrm{CoSb}_{3}$ can lead to either $n$-type or $p$-type conduction $[19,20]$. Sb-enriched samples have $p$-type conduction and Sb-deficient samples will lead to an excess of electrons in the conduction band, showing an $n$-type behavior [21]. Although Sb-vacancy has been tentatively considered to be an important native point defect, density-functional theory calculations performed by Park et al. [14] determined that in Sb-deficient samples, interstitial $\mathrm{Co}\left(\mathrm{Co}_{i}\right)$ is the most stable isolated native point defect. According to Park et al., $\mathrm{Co}_{i}$ is an acceptor-like defect, with a very low formation energy $(0.5 \mathrm{eV})$. This low value can yield a high concentration of $\mathrm{Co}_{i}$ and the formation of a $\mathrm{Co}_{i}$-pair, which is a stable defect at low temperature. The $C o_{i}$-pair is a donor-like defect. However, recent calculations performed by Li et al. [22] determined that a $\mathrm{Co}_{i}$-pair is not expected to form during the process and the breakage of the $\mathrm{Sb}_{4}$ rings induced by $\mathrm{Co}_{i}$ or a $\mathrm{Co}_{i}$-pair promote the formation of lone pairs of electrons in $\mathrm{Sb}$.

The temperature dependent values of the Seebeck coefficient, $S$, showed in Figure $4 \mathrm{~b}$, are negative in the measuring temperature interval, meaning an $n$-type conduction. In $\mathrm{CoSb}_{2.81}$, the non-stoichiometry variation provides extra electrons. The absolute value of $S$ increases up to around $364 \mathrm{~K}$, reaching a maximum of $412 \mu \mathrm{V} / \mathrm{K}$. This value is in good agreement with the value obtained by W.S. Liu et al. [23]. This high obtained absolute value is originated by a lower charge carrier density originated by non-stoichiometry. The typical reported values for the Seebeck coefficient varies from $\sim+100$ to $-400 \mu \mathrm{V} / \mathrm{K}$, at room temperature [23]. It can be observed that the absolute Seebeck coefficient starts to decrease at temperatures above $364 \mathrm{~K}$, which is consistent with the observation made by other authors in $n$-type $\mathrm{CoSb}_{3}$ [11]. The change of the slope is caused by the transition from weak extrinsic conduction, originated by the Sb-deficiency, to the intrinsic conduction.

Goldsmind and Sharp developed the analytical expression $E_{g}=2 \mathrm{e}|S|_{\max } T_{\max }$ relating the bandgap, $E_{g}$, the absolute maximum total Seebeck coefficient, $|S|_{\max }$, and the temperature at which it occurs, $T_{\max }$. From the measured absolute maximum Seebeck coefficient, the calculated value for $E_{g}$ is $0.48 \mathrm{eV}$, which is comparable with the values reported by Wei-Shu et al. [11]. Experimental observations made by Wei-Shu et al. led to the conclusion that $n$-type $\mathrm{CoSb}_{3}$ has a larger energy bandgap than $p$-type $\mathrm{CoSb}_{3}$. According to the defect state model proposed by Park et al. [14], the highest $3 \mathrm{~d}$-like state of $\mathrm{Co}_{i}$ pairs becomes deeper inside the bandgap and, thus, the energy separation between the valence-band maximum and conduction-band minimum becomes higher, increasing the value of the band gap in $n$-type $\mathrm{CoSb}_{3}$.
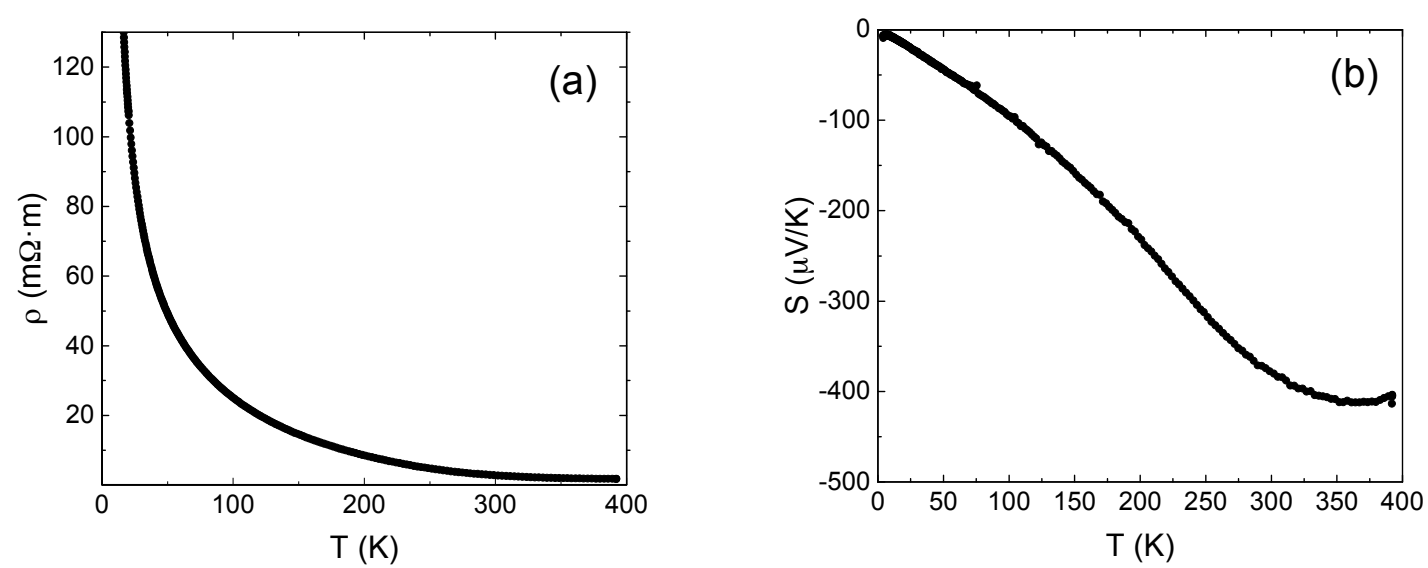

Figure 4. Temperature dependence between 3 and $400 \mathrm{~K}$ of (a) electrical resistivity and (b) Seebeck coefficient. 
According to band structure calculations for $\mathrm{CoSb}_{3}$, the valance band has the first maxima at the $\Gamma$ point and has 12 equivalent second maxima along the $\Gamma \mathrm{N}$ in the $\mathrm{k}$ space. On the other hand, the conduction band has the first minimum at the $\Gamma$ point and has 12 equivalent second minima along the $\Gamma \mathrm{N}$. This structure can be assumed as two valance bands and two conduction bands [24]. One of the valence bands shows a strong non-parabolicity and the scattering of charge carriers are due to non-polar optical phonons, polar optical phonons, ionized impurities, and intervalley transitions. In the remaining bands (one valance band and the two conduction bands) a parabolic dispersion relation is assumed. This parabolic bands are dominated by acoustic-phonon scattering. In a stoichiometric 1:3 compound, it is expected that both, the valance band and the conduction band, contribute with charge carriers. However, the non-stoichiometry of the sample provides extra free electrons, obtaining some extrinsic nature in the sample, where the conduction is primary due to the conduction band at $300 \mathrm{~K}$.

From the experimental Seebeck coefficient value obtained at $300 K, S=-383 \mu \mathrm{V} / \mathrm{K}$, the reduced Fermi level $\eta=-\left(E_{C}-E_{F}\right) / k_{B} T$ can be numerically calculated assuming a single-parabolic conduction band:

$$
S=-\frac{\mathrm{k}_{\mathrm{B}}}{e}\left[\frac{(2+\lambda) F_{1+\lambda}(\eta)}{(1+\lambda) F_{\lambda}(\eta)}-\eta\right]
$$

where $k_{\mathrm{B}}$ is the Boltzmann constant, $e$ is the elementary charge, $\eta$ is the reduced Fermi level, $\lambda$ is a parameter that reflects the dominant scattering mechanism of charge carriers, $h$ the Plank constant, $F_{i}$ the Fermi integral of order $i$ :

$$
F_{i}=\int_{0}^{\infty} \frac{x_{i}}{e^{(x-\eta)}+1} d x
$$

Assuming that $\lambda=0$ for acoustic-phonon scattering which is the generally accepted dominant scattering mechanism for the conduction parabolic band $[25,26]$, the calculated reduced Fermi level was -2.3965 , which means that the Fermi level lies in the bandgap, a characteristic in non-degenerated semiconductors.

Usually, the Hall factor $r_{H}$ is assumed to be equal to 1 , this assumption is valid only in degenerated systems or for energy-independent carrier relaxation time. However, is expected that this factor value will be different from 1 in non-degenerated semiconductors. The value of $r_{H}$ can be estimated by:

$$
r_{H}=\frac{3}{2} F_{\frac{1}{2}}(\eta)\left[\frac{\left(\frac{1}{2}+2 \lambda\right) F_{2 \lambda-\frac{1}{2}}(\eta)}{(1+\lambda)^{2} F_{\lambda}^{2}(\eta)}\right]
$$

assuming again that $\lambda=0$ and taking the previously calculated value of $\eta, r_{H}=1.17$. In non-degenerated semiconductors, $r_{H}$ can vary from 1.18 for scattering by acoustic phonons up to 1.93 for scattering by ionized impurities [27]. The calculated value is really close to 1.18 .

The negative value of Hall coefficient, $R_{h}=-6.1590 \mathrm{~cm}^{3} / \mathrm{C}$, determined from the slope of Hall resistivity variation with magnetic field, from -3 to $3 \mathrm{~T}$, as shown in Figure 5, confirmed that the sample has a $n$-type conduction at $300 \mathrm{~K}$. The linear field dependence of the Hall resistivity corresponds to that of a single type of charge carrier.

In a parabolic dispersion relation, the carrier concentration can be obtained by:

$$
n= \pm \frac{r_{H}}{R_{H} e}
$$

where $e$ is the elementary charge and $r_{H}$ is the Hall factor. Taking the previously calculated value for $r_{H}$ equal to 1.17, the carrier concentration, calculated from the measured Hall coefficient, was $1.19 \times 10^{18} \mathrm{~cm}^{-3}$. 


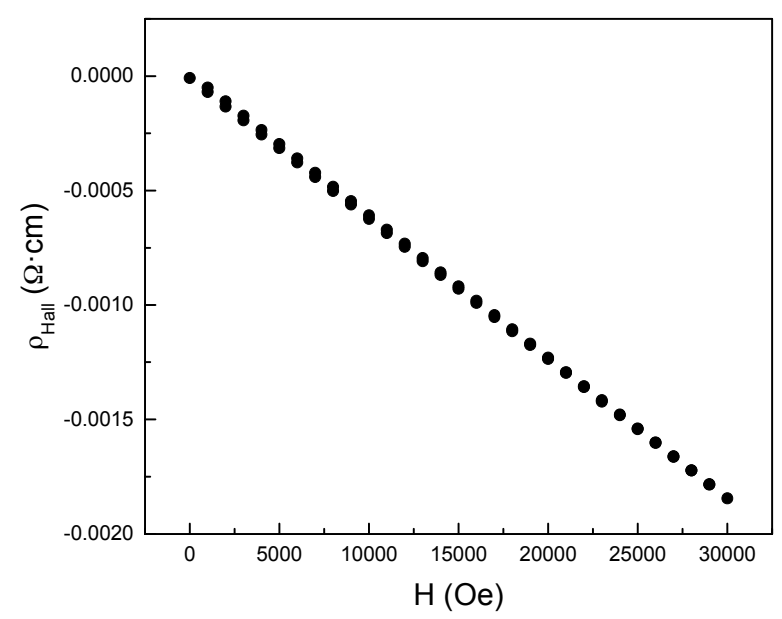

Figure 5. Hall resistivity as a function of magnetic field taken at $T=300 \mathrm{~K}$. At this temperature, the linear magnetic field dependence indicates a single type of charge carrier.

Combining $\eta$ obtained from Seebeck coefficient fitting at $300 \mathrm{~K}$ and $n$, the effective mass of the electron $m^{*}$ can be estimated assuming a parabolic band where acoustic phonon scattering dominates by:

$$
n=4 \pi\left(\frac{2 m^{*} k_{\mathrm{B}} T}{h^{2}}\right)^{3 / 2} F_{1 / 2}(\eta)
$$

obtaining a value of $0.60 m_{0}$, where $m_{0}$ is the free electron mass. Skutterudites are a kind of compound where the hole effective mass is significantly smaller than the electron effective mass [28].

Taking the value of the resistivity as $2.75 \times 10^{-3} \Omega \cdot \mathrm{m}$ at $300 \mathrm{~K}$, Hall mobility $\mu_{H}=R_{H} / \rho$ presented a value of $16.30 \mathrm{~cm}^{2} / \mathrm{V} \cdot \mathrm{s}$ at $300 \mathrm{~K}$. $N$-type $\mathrm{CoSb}_{3}$ mobility are substantially lower compared with the reported values of $p$-type $\mathrm{CoSb}_{3}$ [29], resulting in higher electrical resistivity values.

The Lorenz factor was calculated by the formula:

$$
L=\frac{k_{\mathrm{B}}^{2}}{e^{2}} \frac{(1+\lambda)(3+\lambda) F_{\lambda}(\eta) F_{2+\lambda}(\eta)-(2+\lambda)^{2} F_{1+\lambda}(\eta)^{2}}{(1+\lambda)^{2} F_{\lambda}(\eta)^{2}}
$$

using $\lambda=0$ and the previously calculated $\eta$ value, a Lorenz factor of $1.50 \times 10^{-8} \mathrm{~V}^{2} / \mathrm{K}^{2}$ was obtained. It is important to note that the Lorenz number, as described by the free-electron model, is not an accurate value for most materials and in a given material depends on the detailed band structure, position of the Fermi level and the temperature [30]. The obtained value is significantly lower than the value of the degenerated electron gas, $L_{0}=2.44 \times 10^{-8} \mathrm{~V} / \mathrm{K}^{2}$. In non-degenerated semiconductors, the Lorenz factor decreases up to $40 \%$ of the value of the free electron model [31,32].

\subsection{Thermal Transport Properties}

As shown in Figure 6a, the total thermal conductivity presents a maximum value of $8.24 \mathrm{~W} / \mathrm{K} \cdot \mathrm{m}$ at $50 \mathrm{~K}$. For temperatures lower than the observed maximum, thermal conductivity decreases due to the decreasing of specific heat when the temperature decreases, as proposed in the Debye model. For temperatures higher than the maximum thermal conductivity decreases due to thermally-activated phonon-phonon Umklapp scattering process. The lattice thermal conductivity, $\kappa_{L}$, was obtained by subtracting the electronic contribution, $\kappa_{e}=L_{0} T / \rho$, from the total thermal conductivity, $\kappa_{T}$, where the assumed value for the Lorenz number was the previously calculated one $L_{0}=1.50 \times 10^{-8} \mathrm{~V} / \mathrm{K}^{2}$. The room-temperature lattice thermal conductivity for $\mathrm{CoSb}_{2.81}$ was $3 \mathrm{~W} / \mathrm{K} \cdot \mathrm{m}$ for, which is one of the lowest measured values for this compound and is comparable with the low conductivity obtained by high synthesis pressure [33]. As proposed in the Debye-Callaway model [34], the phonon scattering process can be represented by a relaxation time and this time is derived from a combination 
of relaxation times for scattering by defects, phonon-phonon scattering, boundary scattering, and resonant scattering. The sample is not nanostructured and, thus, the low thermal conductivity in $\mathrm{CoSb}_{2.81}$ observed at room temperature could be related to the scattering of phonons by point defects. This reduction on the lattice thermal conductivity by the presence of defects was predicted by Liu et al. [35].
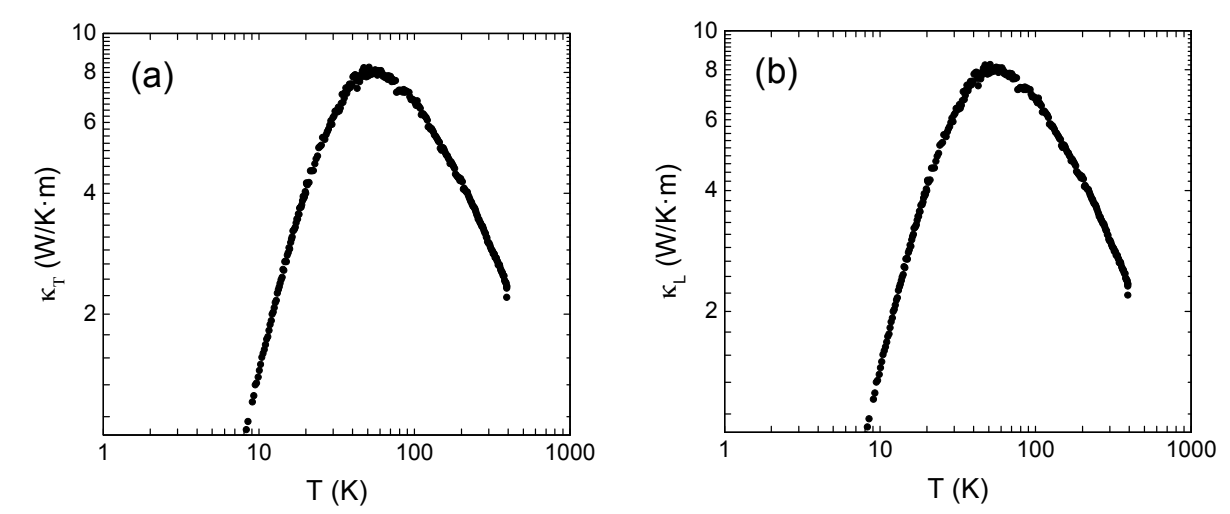

Figure 6. (a) Thermal conductivity and (b) lattice thermal conductivity for $\mathrm{CoSb}_{2.81}$.

\subsection{Figure of Merit}

Figure 7 shows the temperature dependence of the Figure of Merit, ZT, from 3 to $400 \mathrm{~K}$, where a value of $Z T=0.015$ was calculated at the maximum measuring temperature. This value is comparable to other reports measured at the same temperature [34]. The presence of native defects affected the electrical transport, however, restrain the thermal transport.

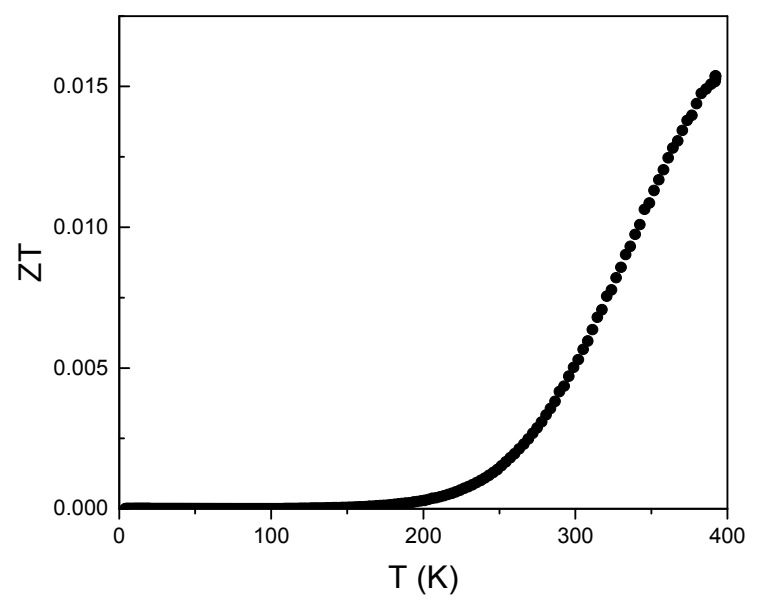

Figure 7. Temperature dependence of the figure of merit between 2 and $400 \mathrm{~K}$.

\section{Conclusions}

A non-linear and a hysteretic behavior in the I-V curves at low temperature were observed. The non-linear behavior was originated from the Poole-Frenkel effect, a field-dependent ionization mechanism, which lowers the Coulomb barrier enhancing the emission of charge carriers. The hysteresis for $T<20 \mathrm{~K}$ was attributed to the drastic decrease of specific heat which produces Joule heating at cryogenic temperature. The $n$-type electrical conduction of the Sb-deficient sample at $300 \mathrm{~K}$ has been attributed to the non-stoichiometry. Within the single parabolic model, and assuming acoustic-phonon scattering, which is the generally accepted dominant scattering mechanism in filled and unfilled skutterudites, some charge transport parameters were calculated from the Seebeck coefficient and Hall measurements performed at $300 \mathrm{~K}$. The numerically calculated reduced Fermi level 
was estimated to be -2.3965 , which means that the Fermi level lies into the band gap, a characteristic in non-degenerated semiconductors. The carrier concentration was about $1.18 \times 10^{18} \mathrm{~cm}^{-3}$. Hall factor, which usually is assumed to be 1 , was calculated to be 1.17 . The Hall mobility of the charge carriers was small and estimated to be $19.11 \mathrm{~cm}^{2} / \mathrm{V} \cdot \mathrm{s}$ caused by the heavy effective mass of the electrons, $0.66 m_{0}$. This low mobility and high effective mass adversely affected the electrical resistivity, obtaining a high resistivity value, $2.75 \times 10^{-3} \Omega \cdot \mathrm{m}$, measured at $300 \mathrm{~K}$. Finally, a calculated value of the Lorenz factor of $1.50 \times 10^{-8} \mathrm{~V}^{2} / \mathrm{K}^{2}$, usually this factor is assumed to be $2.44 \times 10^{-8} \mathrm{~V}^{2} / \mathrm{K}^{2}$ in degenerated semiconductor, however, in non-degenerated semiconductors it depends on the detailed band structure position of the Fermi level and the temperature. The presence of native defects in the non-stoichiometric sample adversely affected the electrical transport properties, however, the thermal conductivity was drastically reduced due phonon scattering by point defects.

Acknowledgments: Authors express their gratitude to CONACYT for the financial support granted for the development of this research through PhD scholarship and to CIMAV, Campus Chihuahua, for the logistical and technical support.

Author Contributions: P.R.R.-G., F.J.R.-G., R.J.S.-H., and M.E.B.-Z. performed synthesis and microstructural characterization. P.R.R.-G., A.F.-A., and C.R.S.-R. measured the physical properties. P.R.R.-G. and J.A.M.-A. designed the experiments, interpreted the results, and wrote the manuscript.

Conflicts of Interest: The authors declare no conflict of interest.

\section{References}

1. Yang, J.; Xi, L.; Qiu, W.; Wu, L.; Shi, X.; Chen, L.; Yang, J.; Zhang, W.; Uher, C.; Singh, D.J. On the tuning of electrical and thermal transport in thermoelectrics: An integrated theory-experiment perspective. NPJ Comput. Mater. 2016, 2, 15015. [CrossRef]

2. Kurosaki, K.; Harnwunggmoung, A.; Yamanaka, S. Thermoelectric properties of $\mathrm{CoSb}_{3}$ based skutterudites filled by group 13 elements. In Nanoscale Thermoelectrics; Wang, X., Wang, M.Z., Eds.; Springer: Cham, Switzerland, 2014; pp. 301-325.

3. Liu, Y.; Li, X.; Zhang, Q.; Zhang, L.; Yu, D.; Xu, B.; Tian, Y. High pressure synthesis of p-type $\mathrm{Ce}_{y} \mathrm{Fe}_{4-x} \mathrm{Co}_{x} \mathrm{Sb}_{12}$ skutterudites. Materials 2016, 9, 257. [CrossRef]

4. Cohn, J.; Nolas, G.; Fessatidis, V.; Metcalf, T.; Slack, G. Glasslike heat conduction in high-mobility crystalline semiconductors. Phys. Rev. Lett. 1999, 82, 779. [CrossRef]

5. Heremans, J.P.; Jovovic, V.; Toberer, E.S.; Saramat, A.; Kurosaki, K.; Charoenphakdee, A.; Yamanaka, S.; Snyder, G.J. Enhancement of thermoelectric efficiency in $\mathrm{PbTe}$ by distortion of the electronic density of states. Science 2008, 321, 554-557. [CrossRef] [PubMed]

6. Pei, Y.; Shi, X.; LaLonde, A.; Wang, H.; Chen, L.; Snyder, G.J. Convergence of electronic bands for high performance bulk thermoelectrics. Nature 2011, 473, 66-69. [CrossRef] [PubMed]

7. Dong, Y.; Puneet, P.; Tritt, T.M.; Nolas, G.S. Crystal structure and high temperature transport properties of Yb-filled p-type skutterudites $\mathrm{Yb}_{x} \mathrm{Co}_{2.5} \mathrm{Fe}_{1.5} \mathrm{Sb}_{12}$. J. Solid State Chem. 2014, 209, 1-5. [CrossRef]

8. Minnich, A.J.; Dresselhaus, M.S.; Ren, Z.F.; Chen, G. Bulk nanostructured thermoelectric materials: Current research and future prospects. Energy Environ. Sci. 2009, 2, 466-479. [CrossRef]

9. Chen, Z.-G.; Han, G.; Yang, L.; Cheng, L.; Zou, J. Nanostructured thermoelectric materials: Current research and future challenge. Prog. Nat. Sci. Mater. Int. 2012, 22, 535-549. [CrossRef]

10. Morelli, D.; Caillat, T.; Fleurial, J.-P.; Borshchevsky, A.; Vandersande, J.; Chen, B.; Uher, C. Low-temperature transport properties of p-type $\mathrm{CoSb}_{3}$. Phys. Rev. B 1995, 51, 9622. [CrossRef]

11. Liu, W.-S.; Zhang, B.-P.; Li, J.-F.; Zhao, L.-D. Effects of $\mathrm{Sb}$ compensation on microstructure, thermoelectric properties and point defect of $\mathrm{CoSb}_{3}$ compound. J. Phys. D Appl. Phys. 2007, 40, 6784-6790. [CrossRef]

12. Zhang, J.; Xu, B.; Yu, F.; Yu, D.; Liu, Z.; He, J.; Tian, Y. Thermoelectric properties of n-type $\mathrm{CoSb}_{3}$ fabricated with high pressure sintering. J. Alloys Compd. 2010, 503, 490-493. [CrossRef]

13. Rodríguez-Carvajal, J. Recent advances in magnetic structure determination by neutron powder diffraction. Phys. B Condens. Matter 1993, 192, 55-69. [CrossRef]

14. Park, C.-H.; Kim, Y.-S. Ab initio study of native point-defects in $\mathrm{CoSb}_{3}$ : Understanding off-stoichiometric doping properties. Phys. Rev. B 2010, 81, 085206. [CrossRef] 
15. Ganichev, S.D.; Ziemann, E.; Prettl, W.; Yassievich, I.N.; Istratov, A.A.; Weber, E.R. Distinction between the poole-frenkel and tunneling models of electric-field-stimulated carrier emission from deep levels in semiconductors. Phys. Rev. B 2000, 61, 10361-10365. [CrossRef]

16. Gutiérrez-D, E.A. Chapter 1-Physics of silicon at cryogenic temperatures-Claeys. In Low Temperature Electronics; Gutierrez-D, E., Deen, J., Claeys, C., Eds.; Academic Press: San Diego, CA, USA, 2001; pp. 1-103.

17. Chen, L.; Kawahara, T.; Tang, X.; Goto, T.; Hirai, T.; Dyck, J.S.; Chen, W.; Uher, C. Anomalous barium filling fraction and n-type thermoelectric performance of $\mathrm{Ba}_{y} \mathrm{Co}_{4} \mathrm{Sb}_{12}$. J. Appl. Phys. 2001, 90, 1864. [CrossRef]

18. Furuyama, S.; Iida, T.; Matsui, S.; Akasaka, M.; Nishio, K.; Takanashi, Y. Thermoelectric properties of undoped p-type $\mathrm{CoSb}_{3}$ prepared by vertical bridgman crystal growth and spark plasma sintering. J. Alloys Compd. 2006, 415, 251-256. [CrossRef]

19. Caillat, T.; Borshchevsky, A.; Fleurial, J.P. Properties of single crystalline semiconducting $\mathrm{CoSb}_{3}$. J. Appl. Phys. 1996, 80, 4442-4449. [CrossRef]

20. Park, C.-H.; Kim, Y.-S. Atomic and electronic structures of co-related point defects in $\mathrm{CoSb}_{3}$. J. Electron. Mater. 2010, 40, 962-966. [CrossRef]

21. Zhang, J.X.; Lu, Q.M.; Liu, K.G.; Zhang, L.; Zhou, M.L. Synthesis and thermoelectric properties of CoSb 3 compounds by spark plasma sintering. Mater. Lett. 2004, 58, 1981-1984. [CrossRef]

22. Li, G.; Bajaj, S.; Aydemir, U.; Hao, S.; Xiao, H.; Goddard, W.A., III; Zhai, P.; Zhang, Q.; Snyder, G.J. P-type Co interstitial defects in thermoelectric skutterudite $\mathrm{CoSb}_{3}$ due to the breakage of $\mathrm{Sb}_{4}$-rings. Chem. Mater. 2016, 28, 2172-2179. [CrossRef]

23. Liu, W.-S.; Zhang, B.-P.; Li, J.-F.; Zhao, L.-D. Thermoelectric property of fine-grained $\mathrm{CoSb}_{3}$ skutterudite compound fabricated by mechanical alloying and spark plasma sintering. J. Phys. D Appl. Phys. 2007, 40, 566-572. [CrossRef]

24. Kajikawa, Y. Strong temperature dependence of the hall factor of p-type $\mathrm{CoSb}_{3}$ : A re-analysis incorporating band nonparabolicity. J. Appl. Phys. 2015, 117, 055702. [CrossRef]

25. Anno, H.; Matsubara, K.; Notohara, Y.; Sakakibara, T.; Tashiro, H. Effects of doping on the transport properties of $\mathrm{CoSb}_{3}$. J. Appl. Phys. 1999, 86, 3780-3786. [CrossRef]

26. Mori, H.; Anno, H.; Matsubara, K. Effect of yb filling on thermoelectric properties of Ge-substituted $\mathrm{CoSb}_{3}$ skutterudites. Mater. Trans. 2005, 46, 1476-1480. [CrossRef]

27. Leszczynski, J.; Ros, V.D.; Lenoir, B.; Dauscher, A.; Candolfi, C.; Masschelein, P.; Hejtmanek, J.; Kutorasinski, K.; Tobola, J.; Smith, R.I.; et al. Electronic band structure, magnetic, transport and thermodynamic properties of In-filled skutterudites $\operatorname{In}_{x} \mathrm{Co}_{4} \mathrm{Sb}_{12}$. J. Phys. D Appl. Phys. 2013, 46, 495106. [CrossRef]

28. Fleurial, J.P.; Caillat, T.; Borshchevsky, A. Skutterudites: An Update. In Proceedings of the 16th International Conference on Thermoelectrics (ICT '97), Dresden, Germany, 26-29 August 1997; pp. 1-11.

29. Mi, J.-L.; Christensen, M.; Nishibori, E.; Kuznetsov, V.; Rowe, D.M.; Iversen, B.B. Multitemperature synchrotron powder diffraction and thermoelectric properties of the skutterudite $\mathrm{La}_{0.1} \mathrm{Co}_{4} \mathrm{Sb}_{12}$. J. Appl. Phys. 2010, 107, 113507. [CrossRef]

30. Lukas, K.C.; Liu, W.S.; Joshi, G.; Zebarjadi, M.; Dresselhaus, M.S.; Ren, Z.F.; Chen, G.; Opeil, C.P. Experimental determination of the lorenz number in $\mathrm{Cu}_{0.01} \mathrm{Bi}_{2} \mathrm{Te}_{2.7} \mathrm{Se}_{0.3}$ and $\mathrm{Bi}_{0.88} \mathrm{Sb}_{0.12}$. Phys. Rev. B 2012, 85, 205410. [CrossRef]

31. Snyder, G.J.; Toberer, E.S. Complex thermoelectric materials. Nat. Mater. 2008, 7, 105-114. [CrossRef] [PubMed]

32. Kim, H.-S.; Gibbs, Z.M.; Tang, Y.; Wang, H.; Snyder, G.J. Characterization of Lorenz number with seebeck coefficient measurement. APL Mater. 2015, 3, 041506. [CrossRef]

33. Deng, L.; Jia, X.; Qin, J.; Wan, Y.; Li, J.; Ma, H. The impact of synthesis pressure on the thermoelectric properties of $\mathrm{CoSb}_{3}$. Mater. Lett. 2013, 93, 219-222. [CrossRef]

34. Callaway, J.; von Baeyer, H.C. Effect of point imperfections on lattice thermal conductivity. Phys. Rev. 1960, 120, 1149-1154. [CrossRef]

35. Liu, Z.; Yang, X.; Chen, G.; Zhai, P. Molecular dynamics study of the influence of Sb-vacancy defects on the lattice thermal conductivity of crystalline $\mathrm{CoSb}_{3}$. Comput. Mater. Sci. 2016, 124, 403-410. [CrossRef]

(C) 2017 by the authors. Licensee MDPI, Basel, Switzerland. This article is an open access article distributed under the terms and conditions of the Creative Commons Attribution (CC BY) license (http:/ / creativecommons.org/licenses/by/4.0/). 